\title{
Approximation Theorems for Random Permanents and Associated Stochastic Processes
}

\author{
Grzegorz A. Rempala \\ Insitute of Mathematics and Its Applications, University of Minnesota \\ and \\ Department of Mathematics, University of Louisville \\ Jacek Wesołowski \\ Wydział Matematyki i Nauk Informacyjnych, Politechnika Warszawska
}

December 29, 2003

\begin{abstract}
The limit theorems for certain stochastic processes generated by permanents of random matrices of independent columns with exchangeable components are established. The results are based on the martingale decomposition of a random permanent function similar to the one known for $U$-statistics and on relating the components of this decomposition to some multiple stochastic integrals.
\end{abstract}

Keywords:Random permanent, permanent process, orthogonal decomposition, invariance principle, Donsker's theorem, elementary symmetric polynomial process, multiple stochastic integral

AMS 2000 Subject Classifications:Primary 60F17,62G20; Secondary 15A15, $15 \mathrm{~A} 52$

Running head: Approximation theorems for random permanents.

\section{Introduction}

Denote by $\mathbb{A}=\left[a_{i j}\right]$ an $m \times n$ real matrix with $m \leq n$. Then a permanent of the matrix $\mathbb{A}$ is defined by

$$
\operatorname{Per} \mathbb{A}=\sum_{\left(i_{1}, \ldots, i_{m}\right):\left\{i_{1}, \ldots, i_{m}\right\} \subset\{1, \ldots, n\}} a_{1 i_{1}} \ldots a_{m i_{m}} .
$$

The permanent function has a long history, having been first introduced by Cauchy in 1812 in his celebrated memoir on determinants. More recently, several problems in statistical mechanics, quantum field theory and chemistry, as well as enumeration problems in combinatorics and linear algebra have been reduced to the computation of a permanent. Unfortunately, the fastest known algorithm for computing a permanent of $n \times n$ matrix runs, as shown by Ryser [15], in $O\left(n 2^{n}\right)$ time. Moreover, strong evidence for the apparent complexity of the problem was provided by Valiant [19] who showed that evaluating a permanent is \#P-complete, even when restricted to $0-1$ matrix. In 
view of these results, the approximation theory for a permanent function in case of a large dimension of the matrix $\mathbb{A}$ has become a very active area of research over past twenty years (for most recent results for both random and non-random settings cf. e.g., Pate[11], Rempała and Wesołowski [13], Forbert and Marx [4], Coelho, and Duffner [3] and references therein).

Let us note that if the entries of $\mathbb{A}$ satisfy $a_{i j}=a_{j}$ for $1 \leq i \leq m$ and $1 \leq j \leq n$, then Per $\mathbb{A}$ reduces, in essence, to an elementary symmetric polynomial of degree $m$ in variables $a_{1}, \ldots, a_{n}$. In probability theory a random permanent function is, therefore, of interest since it can be viewed as a natural generalization of a concept of a random elementary symmetric polynomial, i.e., a $U$-statistic based on the product kernel which asymptotic behavior has been intensely studied (cf. e.g., Székely [17], Móri and Székely [10], van Es and Helmers [20] or Székely and Szeldi [16]). On the other hand, as it has been pointed out in Rempała and Wesołowski [13] for random matrices with all independent entries the permanent function is an example of a $U$-statistic of permanent design, a certain sub-class of incomplete $U$-statistics (cf. e.g., [9]) which enjoys some optimal properties in the sense of the statistical design theory.

In the present work we shall be concerned with the setting where $\mathbb{A}=\mathbb{X}^{(n)}=\left[X_{i j}\right]$ is an $m \times n$ $(m \leq n)$ real random matrix of square integrable components and such that its columns are build from the first $m$ terms of independent identically distributed (iid) sequences $\left(X_{i, 1}\right)_{i \geq 1},\left(X_{i, 2}\right)_{i \geq 1}$, $\ldots,\left(X_{i, n}\right)_{i \geq 1}$ of exchangeable rv's. Note that under these assumptions all entries of the matrix $\mathbb{X}^{(n)}$ are identically distributed, albeit not necessarily independent. For $i, k=1, \ldots, m$ and $j=1, \ldots, n$ we denote $\mu=E X_{i j}, \sigma^{2}=\operatorname{Var} X_{i j}$ and $\rho=\operatorname{Corr}\left(X_{k j}, X_{i j}\right)$. Observe that necessarily $\rho \geq 0$. Assuming in the sequel that $\mu \neq 0$ we denote additionally by $\gamma=\sigma / \mu$ the variation coefficient. Finally, we let $\mathcal{N}$ denote a standard normal rv and $\stackrel{d}{\rightarrow}$ stand for convergence in distribution. In what follows $m=m_{n} \leq n$ is always a non-decreasing sequence. In this notation, we shown herein the following.

\section{Theorem 1 (Approximation theorems for random permanents).}

(i) Suppose $\rho=0$.

$$
\text { If } m / n \rightarrow \lambda>0 \text { as } n \rightarrow \infty \text { then }
$$

$$
\frac{1}{\left(\begin{array}{l}
n \\
m
\end{array}\right) m ! \mu^{m}} \operatorname{Per}\left(\mathbb{X}^{(n)}\right) \stackrel{d}{\rightarrow} \exp \left(\sqrt{\lambda} \gamma \mathcal{N}-\lambda \gamma^{2} / 2\right) .
$$

$$
\text { If } m / n \rightarrow \lambda=0 \text { and } m=m_{n} \rightarrow \infty \text { as } n \rightarrow \infty \text { then }
$$

$$
\sqrt{\frac{n}{m}}\left(\frac{\operatorname{Per}\left(\mathbb{X}^{(n)}\right)}{\left(\begin{array}{l}
n \\
m
\end{array}\right) m ! \mu^{m}}-1\right) \stackrel{d}{\rightarrow} \gamma \mathcal{N} .
$$

(ii) Suppose that $\rho>0$.

$$
\begin{aligned}
& \text { If } m^{2} / n \rightarrow \lambda>0 \text { as } n \rightarrow \infty \text { then } \\
& \qquad \begin{array}{r}
\frac{1}{\left(\begin{array}{l}
n \\
m
\end{array}\right) m ! \mu^{m}} \operatorname{Per}\left(\mathbb{X}^{(n)}\right) \stackrel{d}{\rightarrow} \exp \left(\sqrt{\lambda \rho} \gamma \mathcal{N}-\lambda \rho \gamma^{2} / 2\right) . \\
\text { If } m^{2} / n \rightarrow \lambda=0 \text { and } m=m_{n} \rightarrow \infty \text { as } n \rightarrow \infty \text { then } \\
\frac{\sqrt{n}}{m}\left(\frac{\operatorname{Per}\left(\mathbb{X}^{(n)}\right)}{\left(\begin{array}{l}
n \\
m
\end{array}\right) m ! \mu^{m}}-1\right) \stackrel{d}{\rightarrow} \sqrt{\rho} \gamma \mathcal{N} .
\end{array}
\end{aligned}
$$


This general result extends and gives the common ground to some special cases of the above reported at various times in the literature. See, for instance, van Es and Helmers [20], Korolyuk and Borovskikh [7], Janson [6], and Rempała and Wesołowski [12].

The proof of the result of Theorem 1 relys on the stochasitc integration theory and in fact we find it convenient to prove its functional version instead (from which then Theorem 1 follows as a simple corollary). In order to do so we extend the definition of a permanent function to a stochastic process (in the sequel referred to as "permanent stochastic process" or PSP) in a manner similar to that of extending the concept of an " $U$-statistic" to " $U$-process" (see [9]). That such a construction is possible follows from the orthogonal decomposition of a random permanent introduced in Rempała and Wesołowski [14], similar to the famous Hoeffding decomposition for $U$-statistics (cf, e.g., [9]) and, in fact, being in some sense its natural extension. In order to obtain the final result we first derive a general limit theorem for the elementary symmetric polynomial process (ESPP) based on a triangular array of rowwise independent, zero mean, square integrable random variables (rv's). Our theorem in the special case of the independent, identically distributed (iid) sequence of random variables reduces to the one obtained in Móri and Székely [10] and Kurtz and Protter [8]. For a concise proof, we adapt to our setting the idea of Kurtz and Protter (KP in the sequel) of representing ESPP as a multiple stochastic integral.

The paper is organized as follows. In the reminder of the this section define PSP via orthogonal decomposition of a permanent. In Section 2 we recall some basic facts from the stochastic integration theory and then derive the invariance principle for an ESPP via stochastic integrals convergence theorem of KP. The result is presented as Theorem 2. In Section 3 we relate the results of Section 2 to the asymptotic behavior of the component processes of PSP (Proposition 1). Once this result is established the results for PSP's follow via the truncation-type argument along with the basic properties of the Prohorov distance and the martingale properties of the component processes. These are the main results of the paper and are presented in the last two theorems of Section 3. In particular, from the theorems of Section 3 the approximation theorems of Rempala and Wesolowski quoted above follow immediately.

To define the appropriate random functions related to permanents, let us first note the following useful representation of a random permanent. Namely, it has been proved in [14] that

$$
\frac{\operatorname{Per} \mathbb{X}^{(n)}}{\left(\begin{array}{c}
n \\
m
\end{array}\right) m ! \mu^{m}}=1+\sum_{c=1}^{m}\left(\begin{array}{c}
m \\
c
\end{array}\right) U_{c}^{(m, n)}
$$

where

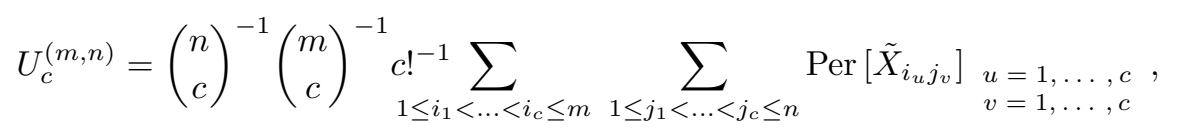

for $\tilde{X}_{i j}=X_{i j} / \mu-1, i=1, \ldots, m, j=1, \ldots, n$. Moreover, the $U_{c}^{(m, n)}$ 's are orthogonal, i.e.,

$$
\operatorname{Cov}\left(U_{c_{1}}^{(m, n)}, U_{c_{2}}^{(m, n)}\right)=0 \quad \text { for } c_{1} \neq c_{2}
$$

as well as

$$
\operatorname{Var} U_{c}^{(m, n)}=\left(\begin{array}{c}
n \\
c
\end{array}\right)^{-1}\left(\begin{array}{c}
m \\
c
\end{array}\right)^{-1} \gamma^{2 c} \sum_{r=0}^{c}\left(\begin{array}{c}
m-r \\
c-r
\end{array}\right) \frac{\rho^{c-r}(1-\rho)^{r}}{r !}
$$


Extending the definition of orthogonal components $U_{c}^{(m, n)}(\mathrm{c}=1,2, \ldots)$ to random functions is straightforward. For any real number $x$ let $[x]$ denote the largest integer less or equal to $x$. Then for any $\mathrm{c}=1,2, \ldots$ and for any $t \in[0,1]$ such that $c \leq[n t]$ let us define

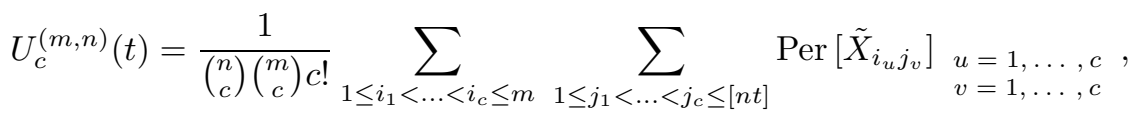

and put $U_{c}^{(m, n)}(t)=0$ if $c>[n t]$.

Now, the decomposition (1) suggests that on the interval $[0,1]$ we may define the stochastic process associated wih $\operatorname{Per}\left(\mathbb{X}^{(n)}\right)$, say $\mathcal{P S P} \mathbb{X}^{(n)}(\cdot)$, by the relation

$$
\mathcal{P S P} \mathbb{X}^{(n)}(t)=\left(\begin{array}{c}
n \\
m
\end{array}\right) m ! \mu^{m}\left[1+\sum_{c=1}^{m}\left(\begin{array}{c}
m \\
c
\end{array}\right) U_{c}^{(m, n)}(t)\right] \quad t \in[0,1] .
$$

It follows from (1) that the trajectory of $\mathcal{P S P} \mathbb{X}^{(n)}(\cdot)$ coincides with a permanent function for $t=1$., i.e.,

$$
\mathcal{P S P} \mathbb{X}^{(n)}(1)=\operatorname{Per}\left(\mathbb{X}^{(n)}\right)
$$

\section{Weak Convergence of the ESPP}

In this section we derive a general result on weak convergence of ESPP based on any rowwise independent triangular array of zero mean, square integrable rv's. The result can be viewed as the extension of Donsker's central limit theorem.

Theorem 2. Let $Y_{l, k}, k=1, \ldots, l, l=1,2, \ldots$, be a double array of square integrable, zero-mean, rowwise independent rv's. Assume that

$$
\begin{gathered}
\lim _{l \rightarrow \infty} \frac{1}{l} \sum_{k=1}^{l} \operatorname{Var} Y_{l, k}=\sigma^{2}, \\
\forall \varepsilon>0 \quad \lim _{l \rightarrow \infty} \frac{1}{l} \sum_{k=1}^{l} E Y_{l, k}^{2} I\left\{\left|Y_{l, k}\right|>\sigma \varepsilon \sqrt{l}\right\}=0 .
\end{gathered}
$$

Then, for any positive integer $c$,

$$
\begin{gathered}
{\left[\left(\frac{S_{[l t]}^{(l)}(1)}{\sqrt{l}}\right)_{t \in[0,1]}, \ldots,\left(\frac{S_{[l t]}^{(l)}(c)}{(\sqrt{l})^{c}}\right)_{t \in[0,1]}\right]^{T} \stackrel{d}{\rightarrow}} \\
\stackrel{d}{\rightarrow}\left[\left(\frac{\sigma t^{1 / 2}}{1 !} H_{1}\left(B_{t} / \sqrt{t}\right)\right)_{t \in[0,1]}, \ldots,\left(\frac{\sigma^{c} c^{c / 2}}{c !} H_{c}\left(B_{t} / \sqrt{t}\right)\right)_{t \in[0,1]}\right]^{T}
\end{gathered}
$$


in the Skorohod space $D_{\mathbf{R}^{c}}([0,1])$, where

$$
S_{[l t]}^{(l)}(j)=\sum_{1 \leq k_{1}<\ldots<k_{j} \leq[l t]} Y_{l, k_{1}} \ldots Y_{l, k_{j}},
$$

is an elementary symmetric polynomial of degree $j, H_{j}$ is the $j$-th Hermite polynomial with leading coefficient equal to one, $j=1,2, \ldots$, and $\mathbf{B}=\left(B_{t}\right)_{t \in[0,1]}$ is the standard Brownian motion.

Remark 1. The asymptotic distribution of $l^{-c / 2} S_{[l t]}^{(l)}(c)$ for fixed $c$ and iid rv's appears in Móri and Székely [10]. The relation (7), again for iid rv's only, appears in KP.

Remark 2. The above theorem can be also viewed as a functional version of the result on elementary symmetric polynomial statistics in Rempała and Wesołowski (2000).

Several different ways of proving the statement (7) are possible. In particular, the approach utilizing the Newton identities along with some properties of elementary symmetric polynomials and the Marcinkiewicz-Zygmund-type law of large numbers could be adapted to the present setting. However, a somewhat simpler proof is also possible, based on a quite general result on the convergence of stochastic integrals, due to KP (see also Jakubowski, Memin and Pagés [5]) . We have chosen the latter approach below since the application of the stochastic integrals theory allows us to present a more concise argument, avoiding many technical details that otherwise would need to be addressed.

In order to proceed we shall recall first some basic facts and definitions. Let $\mathbb{F}=\left(\mathcal{F}_{t}\right)_{t \in[0,1]}$ be a filtration in a probability space $(\Omega, \mathcal{F}, P)$. If $Y=\left(Y_{t}\right)_{t \in[0,1]}$ is a right continuous $\mathcal{F}$ martingale then its quadratic variation process $\left([Y]_{t}\right)_{t \in[0,1]}$ is defined for any $t \in[0,1]$ by

$$
\sum_{k=1}^{n}\left(Y_{u_{k+1}^{(n)}}-Y_{u_{k}^{(n)}}\right)^{2} \stackrel{P}{\rightarrow}[Y]_{t}
$$

where $\left(u_{k}^{(n)}\right)$ is a sequence of partitions of $[0, t]$ such that $\max _{k}\left(u_{k+1}^{(n)}-u_{k}^{(n)}\right) \rightarrow 0$ as $n \rightarrow \infty$. Denote by $D_{\mathbf{R}^{k}}$ the Skorohod space of cadlag functions on $[0,1]$ with values in $\mathbf{R}^{k}$. Let $Y$ be an $\mathbb{F}$ martingale with trajectories in $D_{\mathbf{R}}$ and let $X=\left(X_{t}\right)_{t \in[0,1]}$ be an $\mathbb{F}$ adapted process with trajectories in $D_{\mathbf{R}^{k}}$. Then, following KP we define the stochastic integral as a limit in probability of the Riemann-Stieltjes sums

$$
\int_{0}^{t} X_{s} d Y_{s}=\lim \sum X_{t_{i}(n)}\left(Y_{t_{i+1}^{(n)}}-Y_{t_{i}(n)}\right)
$$

for partitions $\left(t_{i}^{(n)}\right)$ of $[0, t]$ such that $\max \left(t_{i+1}^{(n)}-t_{i}^{(n)}\right)$ converges to zero. The integral exists if the respective limit in probability of the Riemann-Stieltjes sums exists. We will use the notation $\int X d Y$ for the process $\left(\int_{0}^{t} X_{s} d Y_{s}\right)_{t \in[0,1]}$.

The version of the convergence result for stochastic integrals which suits our purposes can be formulated in the following way.

Theorem $3(\mathbf{K P})$. Assume that $(X, Y),\left(X_{1}, Y_{1}\right),\left(X_{2}, Y_{2}\right), \ldots$ are pairs of stochastic processes such that trajectories of the their first components are in $D_{\mathbf{R}^{k}}$ and trajectories of the second components are in $D_{\mathbf{R}}$. Let $Y_{n}$ be a martingale with respect to a filtration $\mathbb{F}_{n}, n=1,2, \ldots$, and $\sup _{n>1} E\left(\left[Y_{n}\right]_{t}\right)<\infty$ for any $t \in[0,1]$, where $\left(\left[Y_{n}\right]_{t}\right)_{t \in[0,1]}$ is the quadratic variation process for the square integrable martingale $Y_{n}$. Assume also that the process $X_{n}$ is $\mathbb{F}_{n}$ adapted, $n=1,2, \ldots$ If $\left(X_{n}, Y_{n}\right)$ converges weakly to $(X, Y)$ in $D_{\mathbf{R}^{k+1}}$ then $\left(X_{n}, Y_{n}, \int X_{n} d Y_{n}\right)$ converges weakly to $\left(X, Y, \int X d Y\right)$ in $D_{\mathbf{R}^{2 k+1}}$. 
We shall use the above result to give a simple proof of our Theorem 2. The idea of representing elementary symmetric polynomials via stochastic integrals comes from Example 3.1 of KP.

Proof. (of Theorem 2) Define for any $k=1,2, \ldots, c$

$$
Y_{n}^{(k)}(t)=\frac{S_{[n t]}^{(n)}(1)}{(\sqrt{n})^{k}}, \quad t \in[0,1] .
$$

Observe that

$$
Y_{n}^{(k)}=\int Y_{n}^{(k-1)} d Y_{n}^{(1)}
$$

Also define

$$
\left(Y_{t}^{(k)}\right)_{t \in[0,1]}=\left(\frac{\sigma^{k} t^{k / 2}}{k !} H_{k}\left(B_{t} / \sqrt{t}\right)\right)_{t \in[0,1]}
$$

Is well known that

$$
Y^{(k)}=\int Y^{(k-1)} d Y^{(1)}
$$

Let $Z_{n}^{(k)}=\left[Y_{n}^{(1)}, \ldots, Y_{n}^{(k)}\right], n=1,2, \ldots$ and let $Z^{(k)}=\left[Y^{(1)}, \ldots, Y^{(k)}\right], k=1,2, \ldots, c$.

To prove that

$$
Z_{n}^{(k)} \stackrel{d}{\rightarrow} Z^{(k)}
$$

in the Skorohod space $D_{\mathbf{R}^{k}}, k=1, \ldots, c$, we use induction with respect to $k$.

The result for $k=1$ follows in view of the conditions (5) and (6) which imply, by the Donsker theorem for triangular arrays (see, e.g. Billingsley [1]), that $Z_{n}^{(1)}=Y_{n}^{(1)} \stackrel{d}{\rightarrow} \mathbf{B}=Y^{(1)}$ in $D_{\mathbf{R}}$. Observe also that the process $Y_{n}^{(1)}$ is a martingale since it is based on the summation of independent rv's. Moreover, since the process $Y_{n}^{(1)}$ just cumulates jumps while remaining constant, for given $n$, in-between jumps, then its quadratic variation is

$$
\left[Y_{n}^{(1)}\right]_{t}=n^{-1} \sum_{k=1}^{[n t]} Y_{n, k}^{2} .
$$

Consequently,

$$
\sup _{n} E\left(\left[Y_{n}^{(1)}\right]_{t}\right)=\sup _{n} \frac{[n t] \sigma^{2}}{n} \leq t \sigma^{2}
$$

Let us also note that all the processes $Y_{n}^{(k)}, k=1, \ldots, c$, are adapted to the same filtration, generated by $Y_{n}^{(1)}, n=1,2, \ldots$, and similarly, the processes $Y^{(k)}, k=1, \ldots, c$, are adapted to the same filtration, generated by the Wiener process $\mathbf{B}$.

Assume now that $Z_{n}^{(k-1)} \stackrel{d}{\rightarrow} Z^{(k-1)}$ in $D_{\mathbf{R}^{k-1}}$. Then, obviously, $\left(Z_{n}^{(k-1)}, Y_{n}^{(1)}\right) \stackrel{d}{\rightarrow}\left(Z^{(k-1)}, Y^{(1)}\right)$ in $D_{\mathbf{R}^{k}}$ since the limiting process is continuous a.s. Consequently, by the KP theorem it follows that

$$
\left(Z_{n}^{(k-1)}, Y_{n}^{(1)}, \int Z_{n}^{(k-1)} d Y_{n}^{(1)}\right) \stackrel{d}{\rightarrow}\left(Z^{(k-1)}, Y^{(1)}, \int Z^{(k-1)} d Y^{(1)}\right)
$$

But the above convergence yields immediately the final result since $\left(Y_{n}^{(1)}, \int Z_{n}^{(k-1)} d Y_{n}^{(1)}\right)=Z_{n}^{(k)}$ and $\left(Y^{(1)}, \int Z^{(k-1)} d Y^{(1)}\right)=Z^{(k)}$. 


\section{Convergence of the component processes}

Recall that by definition

$$
\frac{\mathcal{P S P} \mathbb{X}^{(n)}(t)}{\left(\begin{array}{l}
n \\
m
\end{array}\right) m ! \mu^{m}}=\left[1+\sum_{c=1}^{m}\left(\begin{array}{c}
m \\
c
\end{array}\right) U_{c}^{(m, n)}(t)\right] \quad t \in[0,1] .
$$

We shall first establish the limiting result for $U_{c}^{(m, n)}(\cdot)(c=1,2, \ldots)$.

To this end, let us consider for any $c=1,2, \ldots$ a process $\left(W_{c}^{(n)}(t)\right)$ defined by the following rescaling of $U_{c}^{(m, n)}(t)$.

$$
W_{c}^{(n)}(t)=\left(\begin{array}{c}
n \\
c
\end{array}\right)\left(\begin{array}{c}
m \\
c
\end{array}\right) c ! U_{c}^{(m, n)}(t)=\sum_{1 \leq i_{1}<\ldots i_{c} \leq m} \sum_{\substack{1 \leq j_{1}<\ldots j_{c} \leq[n t] \\
\mathcal{P}}} \mathcal{P} \mathcal{P}\left[\tilde{X}_{i_{u} j_{v}}\right]_{\substack{u=1, \ldots, c \\
v=1, \ldots, c}}
$$

for $t \in[c / n, 1]$ and $W_{c}^{(n)}(t)=0$ for $t \in[0, c / n)$. The reminder of this section is devoted to the proof of the following

Proposition 1. Let $c$ be an arbitrary positive integer. Assume that $m=m_{n} \rightarrow \infty$ as $n \rightarrow \infty$.

$$
\text { If } \rho=0 \text { then }
$$

$$
\begin{aligned}
& {\left[\frac{W_{1}^{(n)}(t)}{\sqrt{m n}}, \ldots, \frac{W_{c}^{(n)}(t)}{(\sqrt{m n})^{c}}\right]_{t \in[0,1]}^{T} \stackrel{d}{\longrightarrow}\left[\frac{\gamma \sqrt{t} H_{1}\left(\frac{B_{t}}{\sqrt{t}}\right)}{1 !}, \ldots, \frac{(\gamma \sqrt{t})^{c} H_{c}\left(\frac{B_{t}}{\sqrt{t}}\right)}{c !}\right]_{t \in[0,1]}^{T} \text { in } D_{\mathbf{R}^{c}} .} \\
& \text { If } \rho>0 \text { then }
\end{aligned}
$$

$$
\left[\frac{W_{1}^{(n)}(t)}{m \sqrt{n}}, \ldots, \frac{W_{c}^{(n)}(t)}{(m \sqrt{n})^{c}}\right]_{t \in[0,1]}^{T} \stackrel{d}{\longrightarrow}\left[\frac{\gamma \sqrt{\rho t} H_{1}\left(\frac{B_{t}}{\sqrt{t}}\right)}{1 !}, \ldots, \frac{(\gamma \sqrt{\rho t})^{c} H_{c}\left(\frac{B_{t}}{\sqrt{t}}\right)}{c !}\right]_{t \in[0,1]}^{T} \quad \text { in } D_{\mathbf{R}^{c}}
$$

Proof. Consider first the case $\rho=0$. For an arbitrary fixed positive integer $c$ define a process $V_{c}^{(n)}$ by

$$
V_{c}^{(n)}(t)=n^{-c / 2} \sum_{1 \leq j_{1}<\ldots<j_{c} \leq[n t]} Y_{n, j_{1}} \ldots Y_{n, j_{c}}
$$

for $t \in[c / n, 1]$, where

$$
Y_{n, j}=m^{-1 / 2} \sum_{i=1}^{m} \tilde{X}_{i, j}, \quad j=1, \ldots, n,
$$

and $V_{c}^{(n)}(t)=0$ for $t \in[0, c / n)$.

Observe that by Theorem 2 the convergence

$$
\left[V_{1}^{(n)}(t), \ldots, V_{c}^{(n)}(t)\right]_{t \in[0,1]} \stackrel{d}{\rightarrow}\left[\frac{\gamma \sqrt{t} H_{1}\left(\frac{B_{t}}{\sqrt{t}}\right)}{1 !}, \ldots, \frac{(\gamma \sqrt{t})^{c} H_{c}\left(\frac{B_{t}}{\sqrt{t}}\right)}{c !}\right]_{t \in[0,1]}^{T}
$$


in $D_{\mathbf{R}^{c}}$ follows as soon as we verify (5) and the Lindeberg condition (6), which in our current setting takes the form

$$
E\left(Y_{n, 1}^{2} I\left(\left|Y_{n, 1}\right|>\sqrt{n} \varepsilon\right)\right) \rightarrow 0
$$

for any $\varepsilon>0$ as $n \rightarrow \infty$. To prove that the condition is satisfied observe first that

$$
E\left(Y_{n, 1}^{2} I\left(\left|Y_{n, 1}\right|>\sqrt{n} \varepsilon\right)\right) \leq \sup _{k \geq 1} E\left(Y_{k, 1}^{2} I\left(Y_{k, 1}^{2}>n \varepsilon^{2}\right)\right) .
$$

Consequently, it suffices to show that the sequence of rv's $\left(Y_{k, 1}^{2}\right)_{k \geq 1}$ is uniformly integrable. To this end, let us note the following.

(i) By a central limit theorem for exchangeable sequences - see, e.g., Taylor et al.[18] - it follows that $Y_{k, 1}^{2}$ converges in distribution to $E\left(\tilde{X}_{1,1}^{2} \mid \mathcal{F}\right) \mathcal{N}^{2}$, where $\mathcal{F}$ is the $\sigma$-algebra of permutable events for the exchangeable sequence $\left(\tilde{X}_{i, 1}\right)_{i \geq 1}$, and $\mathcal{N}$ is a standard normal $\mathrm{rv}$ independent of $\mathcal{F}$, and

(ii) $E\left(E\left(\tilde{X}_{1,1}^{2} \mid \mathcal{F}\right) \mathcal{N}^{2}\right)=E\left(\tilde{X}_{1,1}^{2}\right)=\gamma^{2}$ which, on the other hand, equals $E\left(Y_{1, k}^{2}\right)$ for any $k \geq 1$.

Hence, we conclude that the sequence $\left(Y_{k, 1}^{2}\right)_{k \geq 1}$ is uniformly integrable since it converges in distribution and the corresponding sequence of expectations also converges (all being equal) to the suitable limit. This entails (5) and (6) and thus, via Theorem 2, also (8).

Observe that for any $k=1, \ldots, c$

$$
\frac{W_{k}^{(n)}(t)}{(\sqrt{m n})^{k}}=V_{k}^{(n)}(t)-\frac{R_{k}^{(n)}(t)}{(\sqrt{m n})^{k}}
$$

where $\left(R_{k}^{(n)}(t)\right)$ is a process such that for any $t \in[k / n, 1]$ it is a sum of different products $\tilde{X}_{i_{1}, j_{1}} \ldots \tilde{X}_{i_{k}, j_{k}}$ such that $1 \leq j_{1}<\ldots<j_{k} \leq[n t],\left(i_{1}, \ldots, i_{k}\right) \in\{1, \ldots, m\}^{k}$ and at least one of the indices $i_{1}, \ldots, i_{k}$ in the sequence $\left(i_{1}, \ldots, i_{k}\right)$ repeats. For $t \in[0, k / n)$ we define $R_{k}^{(n)}(t)$ to be equal to zero.

Note that $\left(R_{k}^{(n)}(t)\right)_{t \in[0,1]}$ is a martingale for any fixed $n$, in view of the assumed independence of columns of $\mathbb{X}^{(n)}$. Consequently, by the maximal inequality,

$$
P\left(\sup _{t \in[0,1]} \frac{\left|R_{k}^{(n)}(t)\right|}{(\sqrt{m n})^{k}}>\varepsilon\right) \leq \frac{E\left(R_{k}^{(n)}(1)\right)^{2}}{\varepsilon^{2}(m n)^{k}}=\frac{\operatorname{Var}\left(R_{k}^{(n)}(1)\right)}{\varepsilon^{2}(m n)^{k}} .
$$

Using the fact that $R_{k}^{(n)}(1)$ is a sum of orthogonal products (observe that the covariance of any two of such different products equals zero since the columns are independent and elements in each column have zero correlation) and that the variance of any of such single product equals $\gamma^{2 k}$ while the number of products in $R_{k}^{(n)}(1)$ equals $\left(\begin{array}{l}n \\ k\end{array}\right)\left(m^{k}-\left(\begin{array}{c}m \\ k\end{array}\right) k !\right)$ we obtain

$$
\operatorname{Var}\left(\frac{R_{k}^{(n)}(1)}{(\sqrt{n m})^{k}}\right)=\gamma^{2 k} \frac{\left(\begin{array}{l}
n \\
k
\end{array}\right)\left(m^{k}-\left(\begin{array}{c}
m \\
k
\end{array}\right) k !\right)}{n^{k} m^{k}} \leq \frac{\gamma^{2 k}}{k !} \frac{m^{k}-\left(\begin{array}{c}
m \\
k
\end{array}\right) k !}{m^{k}}=O(1 / m) \rightarrow 0,
$$

as $n \rightarrow \infty$ (since the numerator is of order $m^{k-1}$ while the denominator is of the order $m^{k}$ ). 
Consequently, the $c$-variate process

$$
\left[\frac{R_{1}^{(n)}(t)}{\sqrt{m n}}, \ldots, \frac{R_{c}^{(n)}(t)}{(\sqrt{m n})^{c}}\right]_{t \in[0,1]}^{T} \stackrel{P}{\rightarrow} 0
$$

in $D_{\mathbf{R}^{c}}$ and thus the first result follows immediately by (8) and (9).

Next, let us consider the case $\rho>0$. As above, let us define for any $k=1,2, \ldots$ the process $\left(V_{k}^{(n)}(t)\right)_{t \in[0,1]}$ by

$$
V_{k}^{(n)}(t)=n^{-c / 2} \sum_{1 \leq j_{1}<\ldots<j_{c} \leq[n t]} Y_{n, j_{1}} \ldots Y_{n, j_{c}}
$$

where

$$
Y_{n, j}=\frac{1}{m} \sum_{i=1}^{m} \tilde{X}_{i j}
$$

$j=1,2, \ldots, n$.

Similarly to the previous case, in order to apply the result of Theorem 2, we only need to show that the Lindeberg condition (6) and the condition (5) are satisfied. As above, we shall argue that the sequence $\left(Y_{k, 1}^{2}\right)$ is uniformly integrable. To this end, we observe first that by a version of the law of large numbers for exchangeable sequences (see, e.g., [2], Ch. 7) it follows that

$$
Y_{k, 1}^{2} \stackrel{d}{\rightarrow} E^{2}\left(\tilde{X}_{1,1} \mid \mathcal{F}\right)
$$

where $\mathcal{F}$ is the $\sigma$-algebra of permutable events. Further, by the de Finetti theorem, we have

$$
E\left(E^{2}\left(\tilde{X}_{1,1} \mid \mathcal{F}\right)\right)=E\left(E\left(\tilde{X}_{1,1} \mid \mathcal{F}\right) E\left(\tilde{X}_{2,1} \mid \mathcal{F}\right)\right)=E\left(E\left(\tilde{X}_{1,2} \tilde{X}_{2,1} \mid \mathcal{F}\right)\right)=E\left(\tilde{X}_{1,1} \tilde{X}_{1,2}\right)=\rho \gamma^{2} .
$$

We also note that

$$
E\left(Y_{k, 1}^{2}\right)=\frac{1}{m^{2}}\left[m \gamma^{2}+m(m-1) \rho \gamma^{2}\right] \rightarrow \rho \gamma^{2}, \quad m \rightarrow \infty .
$$

Thus, the sequence $\left(Y_{k, 1}^{2}\right)$ is uniformly integrable and the conditions (5) and (6) of Theorem 2 are satisfied. This allows us to conclude that

$$
\left[V_{1}^{(n)}(t), \ldots, V_{c}^{(n)}(t)\right]_{t \in[0,1]}^{T} \stackrel{d}{\rightarrow}\left[\frac{(\sqrt{\rho t} \gamma)^{1}}{1 !} H_{1}\left(B_{t} / \sqrt{t}\right), \ldots, \frac{(\sqrt{\rho t} \gamma)^{c}}{c !} H_{c}\left(B_{t} / \sqrt{t}\right)\right]_{t \in[0,1]}^{T} .
$$

Similarly to the previous case of uncorrelated components, for any $k=1, \ldots, c$, we have

$$
\frac{W_{k}^{(n)}(t)}{m^{k}(\sqrt{n})^{k}}=V_{k}^{(n)}(t)-\frac{R_{k}^{(n)}(t)}{m^{k}(\sqrt{n})^{k}}
$$

however, note that this time some of the elements of $R_{k}^{(n)}(1)$ are correlated - this is true for the pairs of products originating from exactly the same columns; if at least one column in the pair of products is different then their correlation is zero. Consequently,

$$
\operatorname{Var}\left(\frac{R_{k}^{(n)}(1)}{m^{k} \sqrt{n}^{k}}\right)=\frac{1}{m^{2 k} n^{k}} \sum_{1 \leq j_{1}<\ldots j_{k} \leq n} \operatorname{Var}\left(R_{j_{1}, \ldots, j_{k}}(n)\right)=\frac{\left(\begin{array}{l}
n \\
k
\end{array}\right)}{m^{2 k} n^{k}} \operatorname{Var}\left(R_{1, \ldots, k}(n)\right),
$$


where $R_{j_{1}, \ldots, j_{k}}(n)$ denotes sum of respective products arising from the columns $j_{1}, \ldots, j_{k}$. Since

$$
\left|\operatorname{Cov}\left(\tilde{X}_{i_{1}, j_{1}} \ldots \tilde{X}_{i_{k}, j_{k}}, \tilde{X}_{l_{1}, j_{1}} \ldots \tilde{X}_{l_{k}, j_{k}}\right)\right| \leq \gamma^{2 k}
$$

for any choices of rows $\left(i_{1}, \ldots, i_{k}\right)$ and $\left(l_{1}, \ldots, l_{k}\right)$, we conclude that

$$
\operatorname{Var}\left(R_{1, \ldots, k}(n)\right)<\left(m^{k}-\left(\begin{array}{c}
m \\
k
\end{array}\right) k !\right)^{2} \gamma^{2 k}
$$

Hence, it follows that

$$
\operatorname{Var}\left(\frac{R_{k}^{(n)}(1)}{m^{k}(\sqrt{n})^{k}}\right)<\frac{\left(\begin{array}{l}
n \\
k
\end{array}\right)\left(m^{k}-\left(\begin{array}{c}
m \\
k
\end{array}\right) k !\right)^{2} \gamma^{2 k}}{m^{2 k} n^{k}}<\frac{\gamma^{2 k}}{k !} \frac{\left(m^{k}-\left(\begin{array}{c}
m \\
k
\end{array}\right) k !\right)^{2}}{m^{2 k}} \rightarrow 0
$$

as $n \rightarrow \infty$. As before the process $\left(R_{k}^{(n)}(t)\right)_{t \in[0,1]}$ is a martingale for fixed $n$ and thus once again the maximal inequality yields

$$
\left[\frac{R_{1}^{(n)}(t)}{m \sqrt{n}}, \ldots, \frac{R_{c}^{(n)}(t)}{(m \sqrt{n})^{c}}\right]_{t \in[0,1]}^{T} \stackrel{P}{\longrightarrow} 0 \quad \text { in } D_{\mathbf{R}} .
$$

Now, the second assertion of the theorem follows from (10) and (11) and the proof of Proposition 1 is complete.

\section{Main results}

Having established the result of Proposition 1 in the previous section, we are finally in position to state our two main theorems which are functional versions of the limiting result of Theorem 1. In particular, due to (4) they entail the lattest.

To begin, we consider the case when the entries of matrix $\mathbb{X}$ are uncorrelated.

Theorem 4. Assume that $\rho=0$ and let $\left(B_{t}\right)_{t \in[0,1]}$ denote the standard Brownian motion.

If $m / n \rightarrow \lambda>0$ as $n \rightarrow \infty$ then

$$
\frac{\left(\mathcal{P S P} \mathbf{X}^{(n)}(t)\right)_{t \in[0,1]}}{\left(\begin{array}{c}
n \\
m
\end{array}\right) m ! \mu^{m}} \stackrel{d}{\rightarrow}\left(\exp \left(\sqrt{\lambda} \gamma B_{t}-\lambda t \gamma^{2}\right)\right)_{t \in[0,1]} \quad \text { in } D_{\mathbf{R}}
$$

If $m / n \rightarrow 0$ and $m=m_{n} \rightarrow \infty$ as $n \rightarrow \infty$ then

$$
\sqrt{\frac{n}{m}}\left(\frac{\left(\mathcal{P S P} \mathbf{X}^{(n)}(t)\right)_{t \in[0,1]}}{\left(\begin{array}{c}
n \\
m
\end{array}\right) m ! \mu^{m}}-1\right) \stackrel{d}{\rightarrow} \gamma\left(B_{t}\right)_{t \in[0,1]} \quad \text { in } D_{\mathbf{R}} .
$$

Proof. Consider first the case $\lambda>0$. For any $n$ and any $N$ such that $N<m_{n}$ define a process $\mathbf{S}_{N}^{(n)}=\left(S_{N}^{(n)}(t)\right)_{t \in[0,1]}$ by

$$
S_{N}^{(n)}(t)=1+\sum_{c=1}^{N}\left(\begin{array}{c}
m \\
c
\end{array}\right) U_{c}^{(m, n)}(t)=1+\sum_{c=1}^{N} \frac{(\sqrt{m n})^{c}}{\left(\begin{array}{l}
n \\
c
\end{array}\right) c !} \frac{W_{c}^{(n)}(t)}{(\sqrt{m n})^{c}} .
$$


Observe that by the first assertion of Proposition 1 it follows that

$$
\left(S_{N}^{(n)}(t)\right)_{t \in[0,1]} \stackrel{d}{\rightarrow} \mathbf{G}_{N}=\left(\sum_{c=0}^{N} \frac{\left(\lambda t \gamma^{2}\right)^{c / 2}}{c !} H_{c}\left(B_{t} / \sqrt{t}\right)\right)_{t \in[0,1]}
$$

in $D_{\mathbf{R}}$ as $n \rightarrow \infty$, since for any $c=1,2, \ldots$

$$
\frac{(\sqrt{m n})^{c}}{\left(\begin{array}{l}
n \\
c
\end{array}\right) c !} \rightarrow \lambda^{c / 2} .
$$

Define also a process $\mathbf{T}_{N}^{(n)}=\left(T_{N}^{(n)}(t)\right)_{t \in[0,1]}$ by

$$
T_{N}^{(n)}(t)=\sum_{c=N+1}^{m_{n}}\left(\begin{array}{c}
m \\
c
\end{array}\right) U_{c}^{(m, n)}(t) .
$$

And let $\mathbf{G}_{\infty}=\left(G_{\infty}(t)\right)_{t \in[0,1]}$ be defined by

$$
\left(G_{\infty}(t)\right)_{t \in[0,1]}=\left(\sum_{c=0}^{\infty} \frac{\left(\lambda t \gamma^{2}\right)^{c / 2}}{c !} H_{c}\left(B_{t} / \sqrt{t}\right)\right)_{t \in[0,1]}=\exp \left(\sqrt{\lambda} \gamma B_{t}-\lambda t \gamma^{2}\right)_{t \in[0,1]}
$$

Let $\varrho$ denote the Prokhorov distance between the random elements in $D_{\mathbf{R}}$. Then

$$
\varrho\left(\mathbf{Z}^{(n)}, \mathbf{G}_{\infty}\right) \leq \varrho\left(\mathbf{Z}^{(n)}, \mathbf{S}_{N}^{(n)}\right)+\varrho\left(\mathbf{S}_{N}^{(n)}, \mathbf{G}_{N}\right)+\varrho\left(\mathbf{G}_{N}, \mathbf{G}_{\infty}\right),
$$

where $\mathbf{Z}^{(n)}=\mathbf{S}_{N}^{(n)}+\mathbf{T}_{N}^{(n)}$. Let us note that for any $t \in[0,1]$

$$
\mathcal{P S P} \mathbf{X}^{(n)}(t)=\left(\begin{array}{c}
n \\
m
\end{array}\right) m ! \mu^{m} Z^{(n)}(t)
$$

and that (14) implies $\varrho\left(\mathbf{S}_{N}^{(n)}, \mathbf{G}_{N}\right) \rightarrow 0$ as $n \rightarrow \infty$ for any fixed $N$, as well as, that we have $\varrho\left(\mathbf{G}_{N}, \mathbf{G}_{\infty}\right) \rightarrow 0$ as $N \rightarrow \infty$. Therefore, in order to argue that $\varrho\left(\mathbf{Z}^{(n)}, \mathbf{G}_{\infty}\right) \rightarrow 0$ as $n \rightarrow \infty$ we only need to show that $\varrho\left(\mathbf{Z}^{(n)}, \mathbf{S}_{N}^{(n)}\right)$ tends to zero uniformly in $n$ as $N \rightarrow \infty$.

To this end observe that by the definition of the Prohorov distance

$$
\varrho\left(\mathbf{Z}^{(n)}, \mathbf{S}_{N}^{(n)}\right) \leq \inf \left\{\varepsilon>0: P\left(\sup _{t \in[0,1]}\left|T_{N}^{(n)}(t)\right|>\varepsilon\right) \leq \varepsilon\right\} .
$$

On the other hand, as before, it is easy to see that due to the assumed independence of columns of $\mathbb{X}^{(n)}$ the process $\mathbf{T}_{N}^{(n)}$ is a martingale for fixed $n, N$ and thus, via the maximal inequality and the relations (2) and (3) for $\rho=0$, we have for any $\varepsilon>0$

$$
P\left(\sup _{t \in[0,1]}\left|T_{N}^{(n)}(t)\right|>\varepsilon\right) \leq \varepsilon^{-2} \operatorname{Var} T_{N}^{(n)}(1)=\varepsilon^{-2} \sum_{c=N+1}^{m} \frac{\left(\begin{array}{c}
m \\
c
\end{array}\right) \gamma^{2}}{\left(\begin{array}{c}
n \\
c
\end{array}\right) c !} \leq \varepsilon^{-2} \sum_{c=N+1}^{\infty} \frac{\gamma^{2 c}}{c !}=\varepsilon^{-2} \alpha_{N} \rightarrow 0
$$

as $N \rightarrow \infty$, which entails

$$
\varrho\left(\mathbf{Z}^{(n)}, \mathbf{S}_{N}^{(n)}\right) \leq \sqrt[3]{\alpha_{N}} \rightarrow 0
$$


uniformly in $n$, as $N \rightarrow \infty$ and hence, by (15) that $\varrho\left(\mathbf{Z}^{(n)}, \mathbf{G}_{\infty}\right) \rightarrow 0$ as $n \rightarrow \infty$.

Thus, we conclude that $\mathbf{Z}^{(n)}$ converges weakly to $\mathbf{G}_{\infty}$ in $D_{\mathbf{R}}$.

For the proof in the case $\lambda=0$, let us observe that

$$
\sqrt{\frac{n}{m}} \sum_{c=1}^{m}\left(\begin{array}{c}
m \\
c
\end{array}\right) U_{c}^{(m, n)}(t)=\frac{W_{1}^{(n)}(t)}{\sqrt{m n}}+R^{(m, n)}(t)
$$

where

$$
R^{(m, n)}(t)=\sqrt{\frac{n}{m}} \sum_{c=2}^{m}\left(\begin{array}{c}
m \\
c
\end{array}\right) U_{c}^{(m, n)}(t) .
$$

Observe that due to the independence of columns of the matrix $\mathbb{X}$ the process $\left(R^{(m, n)}(t)\right)$ is a martingale for fixed values of $m, n$. Consequently, by the maximal inequality we get

$$
P\left(\sup _{t \in[0,1]}\left|R^{(m, n)}(t)\right|>\varepsilon\right) \leq \varepsilon^{-2} \operatorname{Var} R^{(m, n)}(1)=\varepsilon^{-2} \frac{n}{m} \sum_{c=2}^{m} \frac{\left(\begin{array}{c}
m \\
c
\end{array}\right) \gamma^{2 c}}{\left(\begin{array}{l}
n \\
c
\end{array}\right) c !} \leq \frac{m}{n} \exp \left(\gamma^{2}\right) \rightarrow 0
$$

and conclude that $\left(R^{(m, n)}(t)\right)$ converges to zero in probability in $D_{\mathbf{R}}$. Hence, the final result follows now directly by Proposition 1.

Remark 3. A quick inspection of the proof of the second part of the theorem reveals that a slight modification of the argument shows the validity of the second part of the hypothesis also for $m$ remaining fixed. In particular, let us note that for $m=1$ we obtain simply Donsker's theorem.

The result of Theorem 4 is complemented with the corresponding one in the case when $\rho>0$.

Theorem 5. Assume that $\rho>0$ and, as before, let $\left(B_{t}\right)_{t \in[0,1]}$ denote the standard Brownian motion.

$$
\begin{aligned}
& \text { If } m^{2} / n \rightarrow \lambda>0 \text { as } n \rightarrow \infty \text { then } \\
& \qquad \frac{\left(\mathcal{P S P} \mathbb{X}{ }^{(n)}(t)\right)_{t \in[0,1]}}{\left(\begin{array}{c}
n \\
m
\end{array}\right) m ! \mu^{m}} \stackrel{d}{\rightarrow} \exp \left(\sqrt{\lambda \rho} \gamma B_{t}-\lambda \rho t \gamma^{2}\right)_{t \in[0,1]} \quad \text { in } D_{\mathbf{R} .} . \\
& \text { If } m^{2} / n \rightarrow 0 \text { and } m=m_{n} \rightarrow \infty \text { as } n \rightarrow \infty \text { then } \\
& \quad \frac{\sqrt{n}}{m}\left(\frac{\left(\mathcal{P S P} \mathbf{X}^{(n)}(t)\right)_{t \in[0,1]}}{\left(\begin{array}{l}
n \\
m
\end{array}\right) m ! \mu^{m}}-1\right) \stackrel{d}{\rightarrow} \sqrt{\rho} \gamma\left(B_{t}\right)_{t \in[0,1]} \quad \text { in } D_{\mathbf{R} .} .
\end{aligned}
$$

Proof. The proof of the result parallels, to large extent, that of Theorem 4. As before, consider first the case $\lambda>0$. For any $n$ and $N$ such that $N<m_{n}$ define a process $\mathbf{S}_{N}^{(n)}=\left(S_{N}^{(n)}(t)\right)_{t \in[0,1]}$ by

$$
S_{N}^{(n)}(t)=1+\sum_{c=1}^{N}\left(\begin{array}{c}
m \\
n
\end{array}\right) U_{c}^{(m, n)}(t)=1+\sum_{c=1}^{N} \frac{(\sqrt{m} n)^{c}}{\left(\begin{array}{l}
n \\
c
\end{array}\right) c !} \frac{W_{c}^{(n)}(t)}{(\sqrt{m} n)^{c}} .
$$

Observe that by the second assertion of Proposition 1 it follows that

$$
\left(S_{N}^{(n)}(t)\right)_{t \in[0,1]} \stackrel{d}{\rightarrow} \mathbf{G}_{N}=\left(\sum_{c=0}^{N} \frac{\left(\lambda \rho t \gamma^{2}\right)^{c / 2}}{c !} H_{c}\left(B_{t} / \sqrt{t}\right)\right)_{t \in[0,1]}
$$


in $D_{\mathbf{R}}$ as $n \rightarrow \infty$, since for any $c=1,2, \ldots$

$$
\frac{(\sqrt{n} m)^{c}}{\left(\begin{array}{l}
n \\
c
\end{array}\right) c !} \rightarrow \lambda^{c / 2}
$$

Define also a process $\mathbf{T}_{N}^{(n)}=\left(T_{N}^{(n)}(t)\right)_{t \in[0,1]}$ by

$$
T_{N}^{(n)}(t)=\sum_{c=N+1}^{m_{n}}\left(\begin{array}{c}
m \\
c
\end{array}\right) U_{c}^{(m, n)}(t) .
$$

And let $\mathbf{G}_{\infty}=\left(G_{\infty}(t)\right)_{t \in[0,1]}$ be defined by

$$
\left(G_{\infty}(t)\right)_{t \in[0,1]}=\left(\sum_{c=0}^{\infty} \frac{\left(\lambda \rho t \gamma^{2}\right)^{c / 2}}{c !} H_{c}\left(B_{t} / t\right)\right)_{t \in[0,1]}=\exp \left(\sqrt{\lambda \rho} \gamma B_{t}-\lambda \rho t \gamma^{2}\right)_{t \in[0,1]} .
$$

As before, if $\varrho$ denotes the Prokhorov distance between the random elements in $D_{\mathbf{R}}$, then

$$
\varrho\left(\mathbf{Z}^{(n)}, \mathbf{G}_{\infty}\right) \leq \varrho\left(\mathbf{Z}^{(n)}, \mathbf{S}_{N}^{(n)}\right)+\varrho\left(\mathbf{S}_{N}^{(n)}, \mathbf{G}_{N}\right)+\varrho\left(\mathbf{G}_{N}, \mathbf{G}_{\infty}\right) .
$$

where $\mathbf{Z}^{(n)}=\mathbf{S}_{N}^{(n)}+\mathbf{T}_{N}^{(n)}$. In order to complete the argument along the lines of the first part of the proof of Theorem 4 we only need to argue that for any $\varepsilon>0$

$$
P\left(\sup _{t \in[0,1]}\left|T_{N}^{(n)}(t)\right|>\varepsilon\right) \rightarrow 0
$$

uniformly in $n$ as $N \rightarrow \infty$. To this end note that again by the martingale property and the maximal inequality as well as by the relations (2) and (3)

$$
\begin{aligned}
P\left(\sup _{t \in[0,1]}\left|T_{N}^{(n)}(t)\right|>\varepsilon\right) & \leq \varepsilon^{-2} \operatorname{Var} T_{N}^{(n)}(1)=\varepsilon^{-2} \frac{n}{m^{2}} \sum_{c=N+1}^{m} \frac{\left(\begin{array}{c}
m \\
c
\end{array}\right) \gamma^{2 c}}{\left(\begin{array}{l}
n \\
c
\end{array}\right)} \sum_{r=0}^{c} \frac{1}{r !}\left(\begin{array}{c}
m-r \\
c-r
\end{array}\right)(1-\rho)^{r} \rho^{c-r} \\
& \leq \varepsilon^{-2} \exp (1) \sum_{c=N+1}^{m}\left(\frac{m^{2}}{n}\right)^{c} \frac{\gamma^{2 c}}{c !}
\end{aligned}
$$

in view of the inequalities $\left(\begin{array}{c}m-r \\ c-r\end{array}\right) \leq\left(\begin{array}{c}m \\ c\end{array}\right)$ and $c ! \frac{\left(\begin{array}{c}m \\ c\end{array}\right)^{2}}{\left(\begin{array}{c}n \\ c\end{array}\right)} \leq\left(\frac{m^{2}}{n}\right)^{c}$ for $0 \leq r \leq c \leq m$. Therefore,

$$
\begin{aligned}
P\left(\sup _{t \in[0,1]}\left|T_{N}^{(n)}(t)\right|>\varepsilon\right) & \leq \varepsilon^{-2} \exp (1) \sum_{c=N+1}^{m}\left(\frac{m^{2}}{n}\right)^{c} \frac{\gamma^{2 c}}{c !} \\
& \leq \varepsilon^{-2} \exp (1) \sum_{c=N+1}^{\infty} \frac{\left(2 \lambda \gamma^{2}\right)^{c}}{c !}=\varepsilon^{-2} \alpha_{N} \rightarrow 0
\end{aligned}
$$

as $N \rightarrow \infty$, and the first part of the theorem follows.

The proof in the case $\lambda=0$, follows similarly to the second part of the proof of Theorem 4 with obvious modifications. Consider

$$
\frac{\sqrt{n}}{m} \sum_{c=1}^{m}\left(\begin{array}{c}
m \\
c
\end{array}\right) U_{c}^{(m, n)}(t)=\frac{W_{1}^{(n)}(t)}{m \sqrt{n}}+R^{(m, n)}(t)
$$


where

$$
R^{(m, n)}(t)=\frac{\sqrt{n}}{m} \sum_{c=2}^{m}\left(\begin{array}{c}
m \\
c
\end{array}\right) U_{c}^{(m, n)}(t)
$$

The process $\left(R^{(m, n)}(t)\right)$ is a martingale for fixed values of $m, n$ and thus

$$
P\left(\sup _{t \in[0,1]}\left|R^{(m, n)}(t)\right|>\varepsilon\right) \leq \varepsilon^{-2} \operatorname{Var} R^{(m, n)}(1) \leq \varepsilon^{-2} \frac{m^{2}}{n} \exp \left(1+\gamma^{2}\right) \rightarrow 0,
$$

follows, in view of

$$
\begin{aligned}
\operatorname{Var} R_{m, n}(1) & =\frac{n}{m^{2}} \sum_{c=2}^{m} \frac{\left(\begin{array}{c}
m \\
c
\end{array}\right) \gamma^{2 c}}{\left(\begin{array}{c}
n \\
c
\end{array}\right)} \sum_{r=0}^{c} \frac{1}{r !}\left(\begin{array}{c}
m-r \\
c-r
\end{array}\right)(1-\rho)^{r} \rho^{c-r} \\
& \leq \exp (1) \frac{n}{m^{2}} \sum_{c=2}^{m}\left(\frac{m^{2}}{n}\right)^{c} \frac{\gamma^{2 c}}{c !} \leq \exp (1) \frac{m^{2}}{n} \sum_{c=2}^{m}\left(\frac{m^{2}}{n}\right)^{c-2} \frac{\gamma^{2 c}}{c !} \\
& \leq \exp (1) \frac{m^{2}}{n} \sum_{c=2}^{m} \frac{\gamma^{2 c}}{c !} \leq \frac{m^{2}}{n} \exp \left(1+\gamma^{2}\right)
\end{aligned}
$$

if only $n$ is large enough to have $m^{2} / n<1$. The result now follows as in the proof of Theorem 4 .

Remark 4. Let us again note that the above result remains valid for $m$ being a fixed constant (see the remark after the proof of Theorem 4).

\section{Acknowledgement}

This work was completed when the first author was visiting the Institute for Mathematics and its Applications at the University of Minnesota. He would like to express his gratitude to the Institute staff and, in particular, its Director, Prof. Douglas G. Arnold, for their hospitality.

\section{References}

[1] Patrick Billingsley. Convergence of probability measures. John Wiley \& Sons Inc., New York, second edition, 1999. A Wiley-Interscience Publication.

[2] Yuan Shih Chow and Henry Teicher. Probability theory. Springer-Verlag, New York, 1978. Independence, interchangeability, martingales.

[3] M. Purificação Coelho and M. Antónia Duffner. On the relation between the determinant and the permanent on symmetric matrices. Linear Multilinear Algebra, 51(2):127-136, 2003.

[4] Harald Forbert and Dominik Marx. Calculation of the permanent of a sparse positive matrix. Comput. Phys. Comm., 150(3):267-273, 2003.

[5] A. Jakubowski, J. Mémin, and G. Pagès. Convergence en loi des suites d'intégrales stochastiques sur l'espace $\mathbf{D}^{1}$ de Skorokhod. Probab. Theory Related Fields, 81(1):111-137, 1989.

[6] Svante Janson. The numbers of spanning trees, Hamilton cycles and perfect matchings in a random graph. Combin. Probab. Comput., 3(1):97-126, 1994. 
[7] V. S. Korolyuk and Yu. V. Borovskikh. Random permanents and symmetric statistics. In Probability theory and mathematical statistics (Kiev, 1991), pages 176-187. World Sci. Publishing, River Edge, NJ, 1992.

[8] Thomas G. Kurtz and Philip Protter. Weak limit theorems for stochastic integrals and stochastic differential equations. Ann. Probab., 19(3):1035-1070, 1991.

[9] A. J. Lee. U-statistics.Theory and practice, volume 110 of Statistics: Textbooks and Monographs. Marcel Dekker Inc., New York, 1990.

[10] T. F. Móri and G. J. Székely. Asymptotic behaviour of symmetric polynomial statistics. Ann. Probab., 10(1):124-131, 1982.

[11] Thomas H. Pate. The best lower bound for the permanent of a correlation matrix of rank two. Linear Multilinear Algebra, 51(3):263-278, 2003.

[12] Grzegorz Rempała and Jacek Wesołowski. Asymptotic behavior of random permanents. Statist. Probab. Lett., 45:149-158, 1999.

[13] Grzegorz Rempala and Jacek Wesolowski. Incomplete $U$-statistics of permanent design. $J$. Nonparametr. Stat., 15(2):221-236, 2003.

[14] Grzegorz A. Rempała and Jacek Wesołowski. Central limit theorems for random permanents with correlation structure. J. Theoret. Probab., 15(1):63-76, 2002.

[15] Herbert John Ryser. Combinatorial mathematics. Published by The Mathematical Association of America, 1963.

[16] G. J. Székely and L. Szeidl. On the limit distribution of random permanents. In Exploring stochastic laws, pages 443-455. VSP, Utrecht, 1995.

[17] Gábor J. Székely. A limit theorem for elementary symmetric polynomials of independent random variables. Z. Wahrsch. Verw. Gebiete, 59(3):355-359, 1982.

[18] Robert L. Taylor, Peter Z. Daffer, and Ronald F. Patterson. Limit theorems for sums of exchangeable random variables. Rowman \& Allanheld Publishers, Totowa, N.J., 1985.

[19] L. G. Valiant. The complexity of computing the permanent. Theoret. Comput. Sci., 8(2):189201, 1979.

[20] A. J. van Es and R. Helmers. Elementary symmetric polynomials of increasing order. Probab. Theory Related Fields, 80(1):21-35, 1988. 